\title{
Unveiling the radio counterparts of two binary AGN candidates: J1108+0659 and J1131-0204 ${ }^{\star}$
}

\author{
M. Bondi ${ }^{1}$, M. A. Pérez-Torres ${ }^{2}$, E. Piconcelli ${ }^{3}$, and $\mathrm{H}$. $\mathrm{Fu}^{4}$ \\ 1 Osservatorio di Radioastronomia-INAF, Bologna, via P. Gobetti 101, 40129 Bologna, Italy \\ e-mail: bondi@ira.inaf.it \\ 2 Instituto de Astrofísica de Andalucía-CSIC, PO Box 3004, 18008 Granada, Spain \\ 3 Osservatorio Astronomico di Roma-INAF, via Frascati 33, 00040 Monte Porzio Catone, Roma, Italy \\ ${ }^{4}$ Department of Physics \& Astronomy, University of Iowa, Iowa City, IA 52245, USA
}

Received 21 December 2015 / Accepted 12 February 2016

\begin{abstract}
The sources SDSS J113126.08-020459.2 and SDSS J110851.04+065901.4 are two double-peaked [O III] emitting active galactic nuclei (AGNs), identified as candidate binary AGNs by optical and near infrared (NIR) observations. We observed the two sources with high resolution Very Long Baseline Interferometry (VLBI) using the European VLBI Network at $5 \mathrm{GHz}$, reduced VLA observations at three frequencies available for one of the sources, and used archival HST observations. For the source SDSS J113126.08-020459.2, the VLBI observations detected only one single compact component associated with the eastern NIR nucleus. In SDSS J110851.04+065901.4, the VLBI observations did not detect any compact components, but the VLA observations allowed us to identify a possible compact core in the region of the north-western optical/NIR nucleus. In this source we find kpc-scale extended radio emission that is spatially coincident to the ultraviolet continuum and to the extended emission narrow line region. The UV continuum is significantly obscured since the amount of extended radio emission yields a star formation rate of about $110 M_{\odot} \mathrm{yr}^{-1}$, which is an order of magnitude larger than implied by the observed ultraviolet emission. Our analysis confirms the presence of only one AGN in the two candidate binary AGNs.
\end{abstract}

Key words. galaxies: active - galaxies: nuclei - galaxies: interactions - radio continuum: galaxies - techniques: interferometric

\section{Introduction}

According to the $\Lambda$ cold dark matter (CDM) hierarchical models of galaxy formation, more massive galaxies are assembled from smaller ones in a series of minor or major merger events (Toomre $\&$ Toomre 1972). Since most massive galaxies host a central supermassive black hole (SMBH), a merger between two galaxies nearly always results in the formation of a merger-remnant galaxy containing two SMBHs (e.g. Kormendy \& Richstone 1995). If sufficient gas accretes onto both SMBHs, each may be visible as an active galactic nucleus (AGN), forming a so-called "dual" or "binary" AGN. Gas and stellar dynamical friction aid the shrinking of the binary AGN, which is gravitationally bound by the stellar bulges with a separation $\gtrsim 1 \mathrm{kpc}$. The duration of this stage of the binary AGN is highly uncertain and depends on the efficiency of the drag from the dynamical friction, but the system will ultimately shrink to form a pc-scale binary SMBH (Begelman et al. 1980; Milosavljević \& Merritt 2001; Madau \& Quataert 2004; Escala et al. 2004; Colpi 2014).

In recent years, moderate resolution optical spectra surveys, such as the Sloan Digital Sky Survey (SDSS Schneider et al. 2010, and references therein) and the DEEP2 Galaxy Redshift Survey (Newman et al. 2013), allowed compilation of catalogues of AGNs with double-peaked [OIII] line emission

\footnotetext{
* FITS files of the reduced images are only available at the CDS via anonymous ftp to cdsarc.u-strasbg. fr (130.79.128.5) or via http://cdsarc.u-strasbg.fr/viz-bin/qcat?]/A+A/588/A102
}

that were identified as dual/binary SMBH candidates (Wang et al. 2009; Smith et al. 2010; Liu et al. 2010b; Shen et al. 2011; Fu et al. 2012). Even though AGN/SMBH pairs are a natural consequence of galaxy mergers and their search has received great attention, evidence for such systems is elusive, so only a few confirmed examples have been found so far (e.g. Junkkarinen et al. 2001; Komossa et al. 2003; Hudson et al. 2006; Rodriguez et al. 2006; Bianchi et al. 2008; Comerford et al. 2009; Bondi \& Pérez-Torres 2010; Piconcelli et al. 2010; Koss et al. 2011; Fu et al. 2011b; Gitti et al. 2013; Deane et al. 2014; Wrobel et al. 2014). A promising approach to identifying real dual AGN, based on the wide-area high resolution VLA radio observations of the SDSS Stripe 82 field (Hodge et al. 2011), has been undertaken by Fu et al. (2015a). This method already confirmed four binary AGN (Fu et al. 2015b), and would eventually yield a sample of $\sim 80$ confirmed binaries that are bright enough for VLBI observations.

The galaxies SDSS J113126.08-020459.2 and SDSS $\mathrm{J} 110851.04+065901.4$ (hereafter J1131-0204 and J1108+0659, respectively) were classified as candidate binary AGNs by Liu et al. (2010b,a), on the basis of spatially resolved double nuclei in the near infrared (NIR) images, whose locations are coincident with the two velocity components of the narrow line emission in the slit spectra (Liu et al. 2010b).

Deep NIR images revealed tidal features and double stellar components separated by $\lesssim 2 \mathrm{kpc}$ in both galaxies (Liu et al. 2010a; Fu et al. 2011a). In each of the two objects, optical slit 
spectroscopy showed two Seyfert-2 like nuclei that were spatially coincident with the stellar components (within 1 arcsec) and with velocity offsets of a few hundred $\mathrm{km} \mathrm{s}^{-1}$ (Liu et al. $2010 b)$. On the other hand, for J1108+0659 integral field spectroscopy showed that the extended narrow line region producing the double-peaked profile is far more complex than originally thought from the longslit spectra, with most of the emission line gas in a region oriented perpendicular to the position angle of the two stellar nuclei (Fu et al. 2012). Finally, the two galaxies were imaged with Chandra and HST (Liu et al. 2013). In J1108+0659 both nuclei were detected in X-rays (albeit the south-east nucleus only at $3.4 \sigma$ level), while no significant X-ray emission was detected in J1131-0204.

In this paper we report on Very Long Baseline Interferometry (VLBI) radio observations at $5 \mathrm{GHz}$ using the European VLBI Network (EVN) for J1108+0659 and J1131-0204, two of the four binary AGN classified by Liu et al. (2010a). For one source, J1108+0659, our analysis is supported by multi-frequency observations carried out by the Karl G. Janski Very Large Array (VLA). For both objects, HST archive observations are available and are used to complement the discussion.

Throughout, we adopt the WMAP concordance $\Lambda$ CDM cosmology with $H_{0}=70 \mathrm{~km} \mathrm{~s}^{-1} \mathrm{Mpc}^{-1}, \Omega_{\text {matter }}=0.27$ and $\Omega_{\text {vacuum }}=0.73$ (Komatsu et al. 2011).

\section{Observations and data analysis}

\subsection{EVN observations}

We observed the radio sources J1131-0204 and J1108+0659 with the European VLBI Network (EVN) at $5 \mathrm{GHz}$ (project code EB050). The observations were conducted in e-VLBI mode (Szomoru 2008) where the data stream is not recorded on disk and later correlated, but is directly transferred to the correlator through fibre links and processed in real time. The maximum data transmission rate per station was $1024 \mathrm{Mbps}$ for a total bandwidth of $128 \mathrm{MHz}$ in both left and right polarization, using two-bit sampling. The observations were carried out on 2011 March 22 in phase-reference mode (e.g. Beasley \& Conway 1995). The nearby calibrators J1136-0330 (at a distance of $1.9^{\circ}$ from $\mathrm{J} 1131-0204$ ) and $\mathrm{J} 1112+0724$ (at a distance of $0.9^{\circ}$ from J1108+0659) were alternated with the target sources, about $1.5 \mathrm{~min}$ for the calibrator and $3.5 \mathrm{~min}$ for the target for a total observing time of about $2 \mathrm{~h}$ on each target source. The following radio telescopes provided useful data: Effelsberg (Germany), Jodrell Bank Mk2 (UK), Medicina (Italy), Onsala (Sweden), Torun (Poland), Yebes (Spain), Westerbork (The Netherlands), Hartebeesthoek (South Africa), and Sheshan (China).

\subsubsection{Data calibration and imaging}

We analysed the correlated data sets using the NRAO Astronomical Image Processing System (AIPS ${ }^{1}$ ) for calibration and imaging purposes. We calibrated the visibility amplitudes using the system temperatures and gain information provided for each telescope. We then performed a channel-based inspection and editing of the data, and corrected the bandpasses using scans on the bright calibrator 4C 39.25. We also applied standard corrections to the phases of the sources in our experiment, including ionosphere corrections (using total electron content measurements publicly available). We corrected the instrumental phase and delay offsets among the baseband converters of each antenna using observations of the calibrators.

\footnotetext{
1 http://www.aips.nrao.edu
}

We imaged and self-calibrated (hybrid mapping procedure) the phase-reference calibrator of each of our target sources, so as to derive the overall amplitude and phase corrections. We then transferred those corrections, scan by scan, to the target sources. Finally, we repeated the fringe-fitting for the phase-reference calibrators, taking their clean component models into account to correct for small residual phases resulting from their structure. We then interpolated the obtained solutions and applied them to the target sources. We imaged the calibrated visibilities of J1131-0204 and J1108+0659 within AIPS. We note that we did not perform any self-calibration of the phases, of the amplitudes, or of the target sources, since the peaks in emission were too faint for such procedures to be applied.

We point out that we kept the averaging integration time to $1 \mathrm{~s}$ and used a maximum channel bandwidth in the imaging of $16 \mathrm{MHz}$, which results in a maximum degradation of the peak response for components far away from the phase centre of less than $2 \%$ at 500 mas and prevents artificial smearing of the images (e.g. Bridle \& Schwab 1999).

In imaging our target sources, we applied the following procedures. When available from high resolution VLA radio images, e.g. J1108+0659, we used the information on the position of the radio components as a prior to search for radio emission on mas scales. For J1131-0204, neither radio images in the literature nor observations in the VLA archive were available. We therefore used the information on the position of the nuclei derived from HST observations (Liu et al. 2013). For both sources, in addition to the fields around the positions of our priors, we imaged 25 additional fields centred on offset positions (where no radio emission would be expected) to test the reliability of a possible detection.

\subsection{VLA observations of $J 1108+0659$}

$\mathrm{J} 1108+0659$ was observed in A-array configuration at $L$ and $C$-bands (on 2011 July 4) and at $X$-bands (on 2011 July 13) under project 11A-175 (PI: Fu). The total bandwidth of the observations was $256 \mathrm{MHz}$, split in two adjacent IFs centred at $1.4 \mathrm{GHz}$ ( $L$-band), $5.0 \mathrm{GHz}$ ( $C$-band), and $8.5 \mathrm{GHz}$ ( $X$ band). The source $3 C 286$ was used as primary flux calibrator, and the compact radio source $\mathrm{J} 1058+0133$ as phase calibrator. The target source $\mathrm{J} 1108+0659$ was observed in $L, C$, and $X$ bands for about 18,27 , and $21 \mathrm{~min}$, respectively. We reduced all three datasets within AIPS using standard procedures.

\subsection{HST archival observations}

We also used HST archival observations of J1131-0204 and $\mathrm{J} 1108+0659$. The two sources were observed using the Wide Field Camera 3 (WFC3) on board the Hubble Space Telescope (HST), programme code GO 12363. The two targets were imaged in the UVIS/F336W $U$-band and IR/F105W (wide $Y$-band) filters. Details of the observations can be found in Liu et al. (2013). We registered the HST astrometry derived from Liu et al. (2013) using the images derived from the VLA data of $\mathrm{J} 1108+0659$. We found that the positions derived by Liu et al. (2013) in the $Y$ band are consistent with the position of the radio component $\mathrm{C}$ at $5.0 \mathrm{GHz}$. Moreover, with the astrometry derived from Liu et al. (2013) the $5 \sigma$ radio source detected at $1.4 \mathrm{GHz}$ at about 9 arcsec SW of J1108+0659 overlaps with an optical galaxy in the $Y$-band image. The absolute astrometric uncertainties of the registered $Y$ and $U$-band images are $\simeq 0.20^{\prime \prime}$ and $\simeq 0.25^{\prime \prime}$, respectively. 


\section{Results and discussion}

\subsection{J1131-0204}

\subsubsection{EVN observations}

Since no high resolution radio image is available for this source, we used the position of the two optical nuclei derived from observations carried out by WFC3 on the HST in the F103W filter (Liu et al. 2013). Using our $5 \mathrm{GHz}$ EVN observations we imaged two 0 !' $25 \times 0$.'25 fields around these positions. We detected a compact feature in the field centred on the eastern nucleus, with a signal-to-noise ratio $(\mathrm{S} / \mathrm{N})$ of about 6.9 (Fig. 1). Our EVN image has a resolution of $15 \times 15$ mas, a peak surface brightness, and a total flux density since the source is unresolved, of $118 \mu \mathrm{Jy} / \mathrm{beam}$. In contrast, we detected no radio emission above a $5 \sigma$ threshold of $85 \mu \mathrm{Jy} /$ beam in the field centred on the western nucleus (even imaging larger field sizes).

Thus, the position of the putative radio core is $\alpha_{\mathrm{J} 2000}=$ $11: 31: 26.088 \delta_{\mathrm{J} 2000}=-02: 04: 59.160$, which is offset with respect to the HST position by less than 0.1 arcsec, so well within the uncertainty of the HST absolute astrometry (Liu et al. 2013). To test the reliability of this radio detection, we imaged 25 additional fields centred at positions that are slightly offset with respect to the first two in order to be used as test blank fields. In each field we searched for the brightness peak in a circular region of radius 0.11 arcsec. We found no brightness peak with $S / N \geq 5.0$ in any of the 25 fields. The mean peak brightness is $60.4 \pm 1.7 \mu \mathrm{Jy} /$ beam where the error is the error on the mean. Therefore, we conclude that the EVN detection of the eastern nucleus of J1131-0204 is significant, whilst we can place only an upper limit for the western nucleus.

\subsubsection{The AGN in $\mathrm{J} 1131-0204$}

This kpc-scale candidate binary AGN is embedded in a massive disk galaxy at $z=0.1463$. Two nuclei, separated by 0.6 arcsec, were detected by NIR imaging (Liu et al. 2010a; Shen et al. 2011). The spatial correspondence between the two stellar continuum peaks and the two [OIII] velocity peaks supported the interpretations that these are two active galaxies (Liu et al. 2010a). On the basis of line diagnostics, both nuclei were optically classified as Type-2 Seyferts, implying that at least one AGN must be present, yet no X-ray emission is detected at the position of the two nuclei above a $3 \sigma$ limit, implying an upper limit on the $\left(0.5-10 \mathrm{keV}\right.$ ) X-ray luminosity of $<10^{41} \mathrm{erg} \mathrm{s}^{-1}$ (Liu et al. 2013).

From our EVN observations we derive a radio luminosity at $5 \mathrm{GHz}$ for the eastern AGN of $L_{R}=6.5 \times 10^{28} \mathrm{erg} \mathrm{s}^{-1} \mathrm{~Hz}^{-1}$. For the western component, assuming as upper limit in the flux density the mean peak brightness of $60.4 \mu \mathrm{Jy}$, gives $L_{R}<$ $3.5 \times 10^{28} \mathrm{erg} \mathrm{s}^{-1} \mathrm{~Hz}^{-1}$. We note that these monochromatic radio luminosities are typical of low luminosity AGN (e.g. Nagar et al. 2005). The radio core detected at $5 \mathrm{GHz}$ is unresolved. Assuming a limit on the source sizes given by the full width at half maximum (FWHM) size of the observing beam, we derive a limit on the brightness temperature given by $T_{\mathrm{b}} \gtrsim 10^{6} \mathrm{~K}$.

We notice that neither of the two possible nuclei of J11310204 is detected in X-rays. Liu et al. (2013) obtained just an upper limit for the $0.5-10 \mathrm{keV}$ luminosity of $10^{41} \mathrm{erg} \mathrm{s}^{-1}$. We estimated the hard X-ray luminosities for both optical nuclei expected on the basis of the X-ray to [OIII] luminosity relation for type 2 AGN reported by Ueda et al. (2015). We derived $L_{2-10 \mathrm{kev}} \simeq 5.8 \times 10^{43} \mathrm{erg} \mathrm{s}^{-1}$ and $\simeq 4.8 \times 10^{43} \mathrm{erg} \mathrm{s}^{-1}$ for the candidate western and eastern nuclei, respectively. The comparison of these estimates with the upper limits inferred by the Chandra

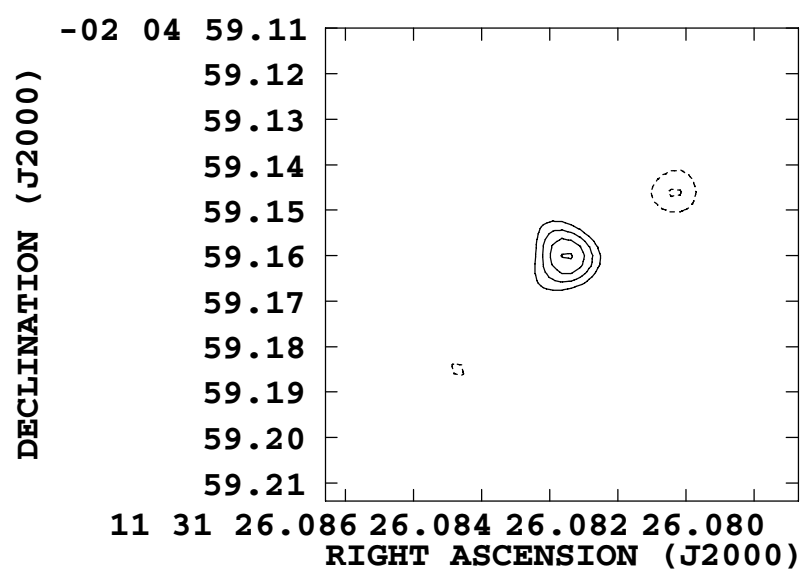

Fig. 1. EVN image at $5 \mathrm{GHz}$ of J1131-0204 restored with a beam size of $15 \times 15$ mas. Peak brightness is $118 \mu \mathrm{Jy} / \mathrm{beam}$ and $\mathrm{rms}$ noise $\sigma$ is $17 \mu \mathrm{Jy}$. Contour levels are drawn at $(-4,-3,3,4,5,6) \times \sigma$.

observation implies a mismatch of a factor of $>100$. This can only be explained by the presence of a very dense, Comptonthick $\left(N_{H} \gg 10^{24} \mathrm{~cm}^{-2}\right)$ obscuring screen along our line of sight to the nucleus. Indeed, a ratio between the observed and intrinsic X-ray luminosity of a factor of $>70-100$ is typically derived for Compton-thick Seyfert galaxies (e.g. Milosavljević \& Merritt 2001). As a merging system, it is likely that a lot of gas and dust have been funnelled into the nuclear region, providing a likely explanation for the apparent buried nature of the AGN.

Using the definition for the radio-loudness parameter of $R_{\mathrm{X}}=v L_{v}(5 \mathrm{GHz}) / L_{\mathrm{X}}$ (Terashima \& Wilson 2003), we derived $\log \left(R_{\mathrm{X}}\right) \simeq-5.2$ for the radio detected eastern nucleus, assuming the hard X-ray luminosity derived from the [OIII] emission. This value characterizes the eastern nucleus of J1131-0204 as a radio-quiet AGN.

Summarizing, from our EVN observations we obtain evidence of just one radio-quiet AGN in J1131-0204, whose position is coincident, within the errors, with the eastern optical nucleus.

\section{2. $J 1108+0659$}

\subsubsection{VLA observations}

In Fig. 2 we display the contour maps of the images obtained by the VLA observations at $1.4,5.0$ e $8,5 \mathrm{GHz}$. At $1.4 \mathrm{GHz}$ (top panel), the source is slightly resolved with a resolution of $2.30 \times 1.38$ arcsec, and is fitted by a Gaussian component with a deconvolved FWHM size of $1.9 \times 1.0$ arcsec in position angle $16^{\circ}$. The fitted total flux is $9.82 \pm 0.15 \mathrm{mJy}$, consistent with the flux of 9.84 mJy measured in the FIRST survey (White et al. 1997).

At sub-arcsecond resolution, the $5.0 \mathrm{GHz}$ and $8.5 \mathrm{GHz}$ images (middle and bottom panels in Fig. 2) show interesting and new features. At the intermediate frequency (resolution $0.46 \times$ 0.37 arcsec), the source morphology is mainly characterized by three components. The brightest central component (labelled as $\mathrm{C}$ ) is elongated at position angle $113^{\circ}$ and can be fit either by a single extended component or by two more compact components embedded in some diffuse emission, mainly on the northwestern side. The total flux of component $\mathrm{C}$ is about $1.4 \mathrm{mJy}$. A tail of extended emission (labelled as B) is detected in the south-western direction from the central component. This emission extends for more than 1 arcsec from component $\mathrm{C}$ and 

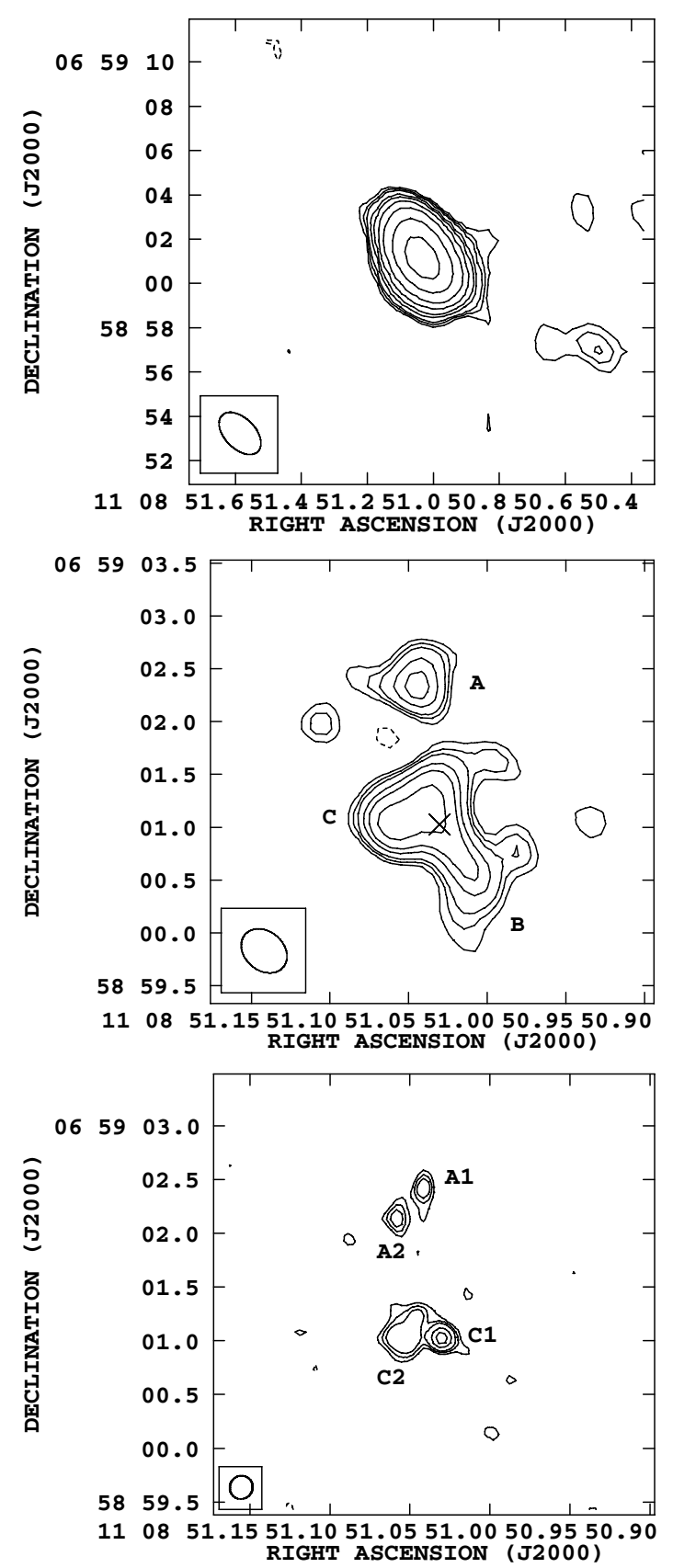

Fig. 2. VLA images of J1108+0659. Top panel: image at $1.4 \mathrm{GHz}$, beam size is $2.30 \times 1.38$ arcsec, peak flux is $5.82 \mathrm{mJy} / \mathrm{beam}$, and $\mathrm{rms}$ noise $\sigma=$ $0.040 \mathrm{mJy}$. Middle panel: image at $5.0 \mathrm{GHz}$, beam size is $0.46 \times 0.37$ arcsec, peak flux is $0.41 \mathrm{mJy} / \mathrm{beam}, \mathrm{rms}$ noise $\sigma=0.020 \mathrm{mJy}$. Bottom panel: image at $8.5 \mathrm{GHz}$, beam size is $0.22 \times 0.21$ arcsec, peak flux is $0.20 \mathrm{mJy} /$ beam, rms noise $\sigma=0.017 \mathrm{mJy}$. For all three images, contour levels are $(-3,3,4,5,7.5,10,15,25,50,100) \times \sigma$. Labels identify components discussed in the text, the cross in the middle panel is drawn at the position of component $\mathrm{C} 1$.

accounts for $\sim 0.5 \mathrm{mJy}$. On the northern side, a resolved component (deconvolved FWHM size of $0.38 \times 0.25$ arcsec in position angle $133^{\circ}$ ) with a total flux density of $0.38 \mathrm{mJy}$ is detected (labelled A). Accounting for the extended emission between the three major components, the total flux density at $5.0 \mathrm{GHz}$ is $2.6 \pm 0.1 \mathrm{mJy}$.

At the highest resolution $(0.22 \times 0.21 \mathrm{arcsec})$ of the $8.5 \mathrm{GHz}$ image, the extended emission of the south-western tail is resolved out. The central component $\mathrm{C}$ is resolved into a compact core, labelled $\mathrm{C} 1$, detected at position $\alpha_{\mathrm{J} 2000}=11: 08: 51.030$ $\delta_{\mathrm{J} 2000}=06: 59: 01.03$ and a resolved component $\mathrm{C} 2$. The core $\mathrm{C} 1$ is only slightly resolved with a fitted FWHM size of $0.14 \times$ 0.04 arcsec in position angle $52^{\circ}$. The northern component detected at $5.0 \mathrm{GHz}$ is resolved in two components, A1 and A2, separated by 0.39 arcsec (about $1.2 \mathrm{kpc}$ ) in position angle $140^{\circ}$, consistent with the single component fit at $5.0 \mathrm{GHz}$. The two components $\mathrm{A} 1$ and $\mathrm{A} 2$ are both slightly resolved in the NS direction.

\subsubsection{EVN observations}

We used the information on the position of the radio components $\mathrm{A} 1, \mathrm{~A} 2$, and $\mathrm{C} 1$ from the $8.5 \mathrm{GHz}$ VLA image as a prior to search for radio emission in our EVN observations. We detected no radio emission associated to any of these components above a significant level of confidence $(\geq 5 \sigma)$. We therefore put a limit on the flux density of the mas-scale core of $\sim 85 \mu \mathrm{Jy}$ at $5 \mathrm{GHz}$. There are obvious possible reasons for not having detected $\mathrm{C} 1$, the most compact VLA component in our EVN observations. It is clear from the radio morphology and fitted size that, at the resolution of the VLA, the component $\mathrm{C} 1$ can still be affected by emission from the eastern lobe, $\mathrm{C} 2$, and/or from a jet component feeding the lobe on scales of several tens of mas. Therefore it is possible that we did not detect a core component on the mas scale due to the sensitivity limit of our EVN observations. Observations filling the gap in resolution between the $8.5 \mathrm{GHz}$ VLA data and our EVN observations at $5 \mathrm{GHz}$ would help to solve this issue.

\subsubsection{Radio spectral index}

We derived radio spectral index information for J1108+0659 using the VLA data at $5.0 \mathrm{GHz}$ and $8.5 \mathrm{GHz}$, as the resolution of the $1.4 \mathrm{GHz}$ image is too poor to derive the spectral index of different radio components. To this end, we imaged the two data sets with the same resolution $(0.41 \times 0.41 \mathrm{arcsec}$; see Fig. 3$)$.

Then, we combined the images at the two frequencies to produce a spectral index image and a spectral index error image. We identified three main components for which we extract the spectral index information presented in Table 1. For each component we list the mean spectral index (the mean of the spectral index values over the region covered by the component) and the dispersion of the spectral index values with respect to the mean $(\sigma)$ and the mean error, i.e. the mean from the spectral index error image in the region $\left(\sigma_{\mathrm{M}}\right)$. The northernmost radio component (A) contains both components $\mathrm{A} 1$ and $\mathrm{A} 2$, which cannot be resolved at this resolution. The spectral index in this region is very smooth and uniform $(\sigma=0.07)$ throughout the region, implying that both $\mathrm{A} 1$ and $\mathrm{A} 2$ have similar spectral properties. The mean spectral index of this component is $0.4 \pm 0.2$, where the error is the mean error of the derived spectral index values in this region. This radio component is located in the northern tail of star formation activity.

The central component $(\mathrm{C})$ is clearly extended at both frequencies (Fig. 3). Its is worth noting how the shape, in particular the position angle, of this component changes with frequency. At $5.0 \mathrm{GHz}$, the component is extended with about the same position angle of the separation between the two optical nuclei and the position angle of the extended $U$-band emission starting from the north-west optical nucleus that traces recent star formation (Fig. 4). We also note how the $5.0 \mathrm{GHz}$ radio emission 

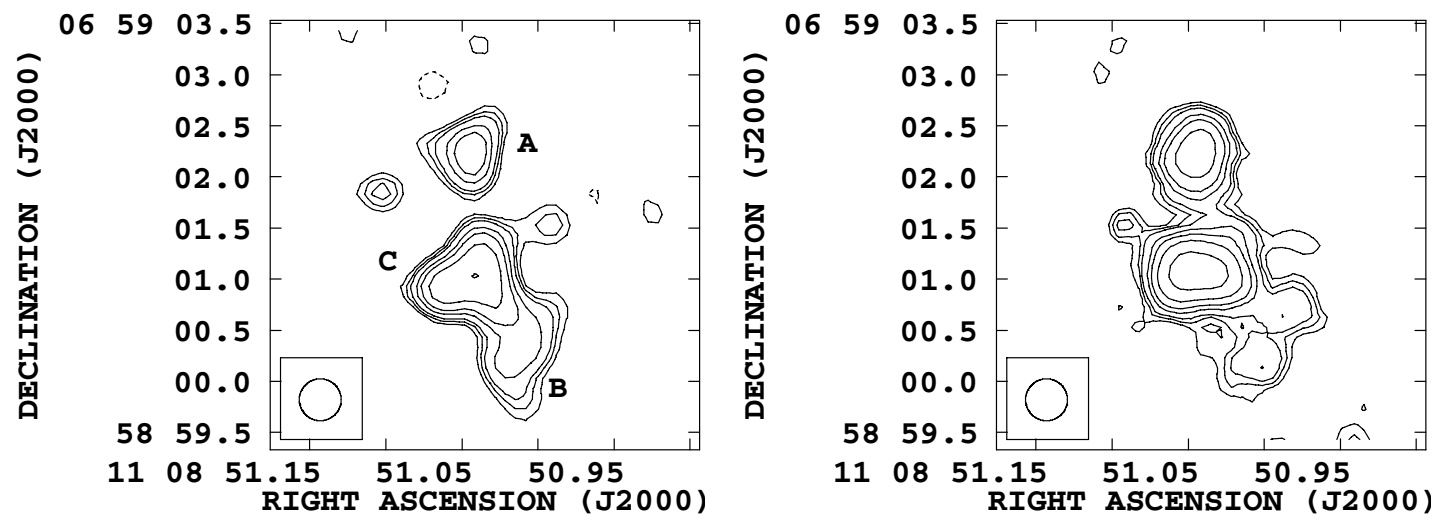

Fig. 3. VLA images of J1108+0659 used for spectral index analysis. Left panel: image at $5.0 \mathrm{GHz}$, beam size is $0.41 \times 0.41$ arcsec, peak flux is $0.50 \mathrm{mJy} /$ beam, rms noise $\sigma=0.025 \mathrm{mJy}$. Right panel: image at $8.5 \mathrm{GHz}$, beam size is $0.41 \times 0.41 \mathrm{arcsec}$, peak flux is $0.34 \mathrm{mJy} / \mathrm{beam}$, rms noise $\sigma=0.015 \mathrm{mJy}$. For all three images, contour levels are $-3,3,4,5,7.5,10,15,25,50,100 \times \sigma$.

Table 1. Mean radio spectral index, $\alpha\left(S_{v} \propto v^{-\alpha}\right)$ of selected regions in $\mathrm{J} 1108+0659$.

\begin{tabular}{llll}
\hline \hline Comp. & $\alpha$ & $\sigma$ & $\sigma_{\mathrm{M}}$ \\
& & & \\
\hline $\mathrm{A}$ & 0.4 & 0.07 & 0.2 \\
$\mathrm{C}$ & 0.8 & 0.5 & 0.2 \\
$\mathrm{~B}$ & 1.7 & 0.8 & 0.5 \\
\hline
\end{tabular}

Notes. $\sigma$ is the dipersion of $\alpha$, and $\sigma_{\mathrm{M}}$ is the average error on $\alpha$.

follows a filament of star formation activity in the north-west region of this component.

At higher frequency, the position angle of component $\mathrm{C}$ flips by about $40^{\circ}$, most likely due to the emergence of a component with a flatter spectrum. This emerging component is the candidate radio nucleus $\mathrm{C} 1$, and it becomes the brightest component in the high resolution $8.5 \mathrm{GHz}$ image (see Fig. 2). The average spectral index in region $\mathrm{C}$ is steep, but not homogenous, and the rather large dispersion $(\sigma=0.5)$ is due to large variations in the spectral index across the component, owing to the superposition of components with different spectral shapes.

The southern component B has a very steep spectral index $\alpha=1.5$ with $\sigma=0.8$. In this region the large dispersion is mainly due to the large errors associated to the spectral index values. The radio emission is clearly associated to the extended $U$-band emission-tracing region of star formation activity. Finally, we made an attempt to estimate the spectral index of component $\mathrm{C} 1$. Component $\mathrm{C} 1$ has a total flux of about $200 \mu \mathrm{Jy}$ and a deconvolved FWHM of $0.14 \times 0.04$ arcsec in position angle $52^{\circ}$. This component is not prominent in the $5.0 \mathrm{GHz}$ image, since it is embedded in the more diffuse emission of component C. This makes it challenging to derive the radio spectral index of this component. We estimated the radio spectral index of $\mathrm{C} 1$ using flux densities extracted from the same region at $8.5 \mathrm{GHz}$ and $5.0 \mathrm{GHz}$ using images at the highest (but different) resolutions, and obtained $\alpha_{\mathrm{C} 1} \simeq 0.3 \pm 0.2$. The uncertainty in the spectral index value is due to the estimate of the contribution of the diffuse emission to the flux density measured at 5.0 GHz.

\subsubsection{AGN and/or star formation emission in J1108+0659}

Near-infrared imaging revealed two stellar nuclei, which are separated by $\simeq 0.7$ arcsec in position angle $141^{\circ}$ and completely enshrouded by the extended narrow line emission (Liu et al. 2010a; Fu et al. 2011a; Liu et al. 2013). The extended narrow line region (ENLR) is centred on the double stellar components and extends for several arcseconds in position angle $\theta_{\text {ENLR }} \simeq 0^{\circ}$ (Fu et al. 2012). All the line diagnostics are consistent with the presence and dominance of at least one type-2 AGN with Seyfert-like luminosity (Liu et al. 2010b,a; Fu et al. 2011a, 2012; Shirazi \& Brinchmann 2012). Although with limited confidence, both stellar nuclei are detected in the X-ray Chandra observations (Liu et al. 2013). The hardness ratio derived for the north-west nucleus is consistent with a steep spectral index of $\Gamma \simeq 2.4$, which is typically associated to starburst galaxies (Dahlem et al. 1998). This hypothesis is also supported by the estimated soft/hard X-ray luminosity of $4 \times 10^{41} \mathrm{erg} \mathrm{s}^{-1}$, which is in the starburst range. In contrast, the south-east nucleus exhibits both the X-ray flat spectral shape and luminosity of an obscured AGN, although it was detected at a $3.4 \sigma$ level. Specifically, Liu et al. (2013) measured a column density of $N_{H} \simeq 3 \times 10^{22} \mathrm{~cm}^{-2}$, which implies an X-ray de-absorbed luminosity of $\simeq 1.2 \times 10^{42} \mathrm{erg} \mathrm{s}^{-1}$, suggesting the presence of an AGN.

On the other hand, the UV-continuum is centred on the northwest stellar component and extends for a few arcseconds along the same position angle of the [OIII] line emission. Using the $\mathrm{X}$-ray to [OIII] luminosity relation for type 2 AGN reported by Ueda et al. (2015), we derive hard X-ray luminosities of $1 \times 10^{44} \mathrm{erg} \mathrm{s}^{-1}$ and $1.6 \times 10^{43} \mathrm{erg} \mathrm{s}^{-1}$ for the north-west and south.east nucleus, respectively. While the value for the southeast nucleus is broadly consistent with that meaured by Liu et al. (2013), there is a mismatch of a factor $\sim 100$ for the north-west component.

$\mathrm{C} 1$, which is the most compact radio component and has the flattest spectral index, is the best candidate for hosting the radio nucleus of $\mathrm{J} 1108+0659$, although the offset of the position of $\mathrm{C} 1$ with respect to the HST and X-ray position of the northwest optical/NIR core ( 0.29 and 0.23 , respectively) may cast some doubts. Still, this value is comparable to the uncertainty in the HST astrometry. In addition, the relative alignment of the radio extended emission with the HST $U$-band emission is rather good, and it would be worsened if the north-west optical core would be shifted to the position of $\mathrm{C} 1$. Figure 4 shows the offset between component $\mathrm{C} 1$ and the north-west UV/NIR core. 
Components A1 and A2 have a slightly steeper spectral index than $\mathrm{C} 1$, are less compact, and are distant ( $>1$ arcsec) from both stellar optical/NIR nuclei, and therefore they do not seem to be good candidates for hosting the radio core of an AGN. Moreover, they are located at the northern peak of the [OIII] narrow line emission (see Fig.3 in Fu et al. 2012), in a region where intense star formation is occurring.

Summarising, from our VLA observations, we find some evidences that component $\mathrm{C} 1$ is the radio nucleus of the AGN: it has the flattest radio spectrum and is the most compact component in our highest frequency image. On the other hand, we note that the presence of a mas-scale compact core is not confirmed by our EVN observations, albeit this could be explained by lack of sensitivity, and that the position of component $\mathrm{C} 1$ is offset with respect to the HST and X-ray Chandra position of the eastern core. Further high sensitivity data, with an angular resolution filling the gap between the $8.5 \mathrm{GHz}$ VLA and $5 \mathrm{GHz}$ EVN observations, are needed to unambiguously confirm the presence of a radio AGN in $\mathrm{J} 1108+0659$.

The extended UV emission is not the only evidence for intense star formation in $\mathrm{J} 1108+0659$. The source is associated to the IRAS Faint Source Catalog galaxy F11062+0715 (Moshir et al. 1992; Hou et al. 2009). While the association has been claimed [by others] as tentative due to the large offset between the IRAS and optical positions $(\simeq 9 \operatorname{arcsec})$, we note that the $1 \sigma$ ellipse error for F11062+0715 is $27 \times 7$ arcsec, and therefore the association seems secure within the uncertainties of the IRAS position. F11062+0715 has ULIRG-like luminosity with a total IR luminosity $L_{\mathrm{IR}}(1-1000 \mu \mathrm{m})=12.01$ (Hou et al. 2009).

An overplot of the radio contours at $5.0 \mathrm{GHz}$ over the $U$-band HST image is shown in Fig. 4. The $U$-band image reveals intense star formation activity in the north-west nuclear region, while the south-east nucleus is undetected, indicating a very low level of star formation around this nucleus, or that the star formation is highly obscured (Liu et al. 2013).

The extended radio emission is co-spatial with the $U$-band UV emission and with the [OIII] line emission, supporting intense star formation as a common origin for the radio and UV continuum. We notice that the different indicators of star formation rate (SFR) give different results. Indeed, on the basis of the $U$-band luminosity, Liu et al. (2013) derived a star formation rate $S F R_{\mathrm{UV}} \simeq 10 M_{\odot} \mathrm{yr}^{-1}$. On the other hand, using the total IR luminosity yields a $S F R_{\mathrm{IR}} \simeq 100 M_{\odot} \mathrm{yr}^{-1}$, an order of magnitude greater than derived from the $U$-band emission. To derive the star formation rate from the radio emission, we need to separate the synchrotron emission related to the AGN (core and lobe/jet emission) from what is associated with star formation activity. For simplicity, we assume here that component $\mathrm{C}$ (including $\mathrm{C} 1$ ) is produced by AGN-related radio emission. The total flux density at $5.0 \mathrm{GHz}$ of components $\mathrm{A}$ and $\mathrm{B}$ is about $0.9 \mathrm{mJy}$. Using an average spectral index $\alpha=0.8$, we obtain a radio luminosity at $1.4 \mathrm{GHz}$ associated to star formation $L_{\mathrm{SF}} \simeq 2.2 \times 10^{30} \mathrm{erg} \mathrm{s}^{-1} \mathrm{~Hz}^{-1}$ corresponding to a $S F R_{1.4 \mathrm{GHz}} \simeq 110 M_{\odot} \mathrm{yr}^{-1}$ consistent with the rate derived from the total infrared luminosity. We conclude that a large part of the UV-emission must be obscured, yielding a SFR estimate in this band an order of magnitude less than derived from measurements unaffected by dust absorption.

\section{Summary}

We have reported on EVN observations at $5 \mathrm{GHz}$ of two dual AGN candidates J1131-0204 and J1108+0659 (supported by multifrequency VLA observations for this source). The main
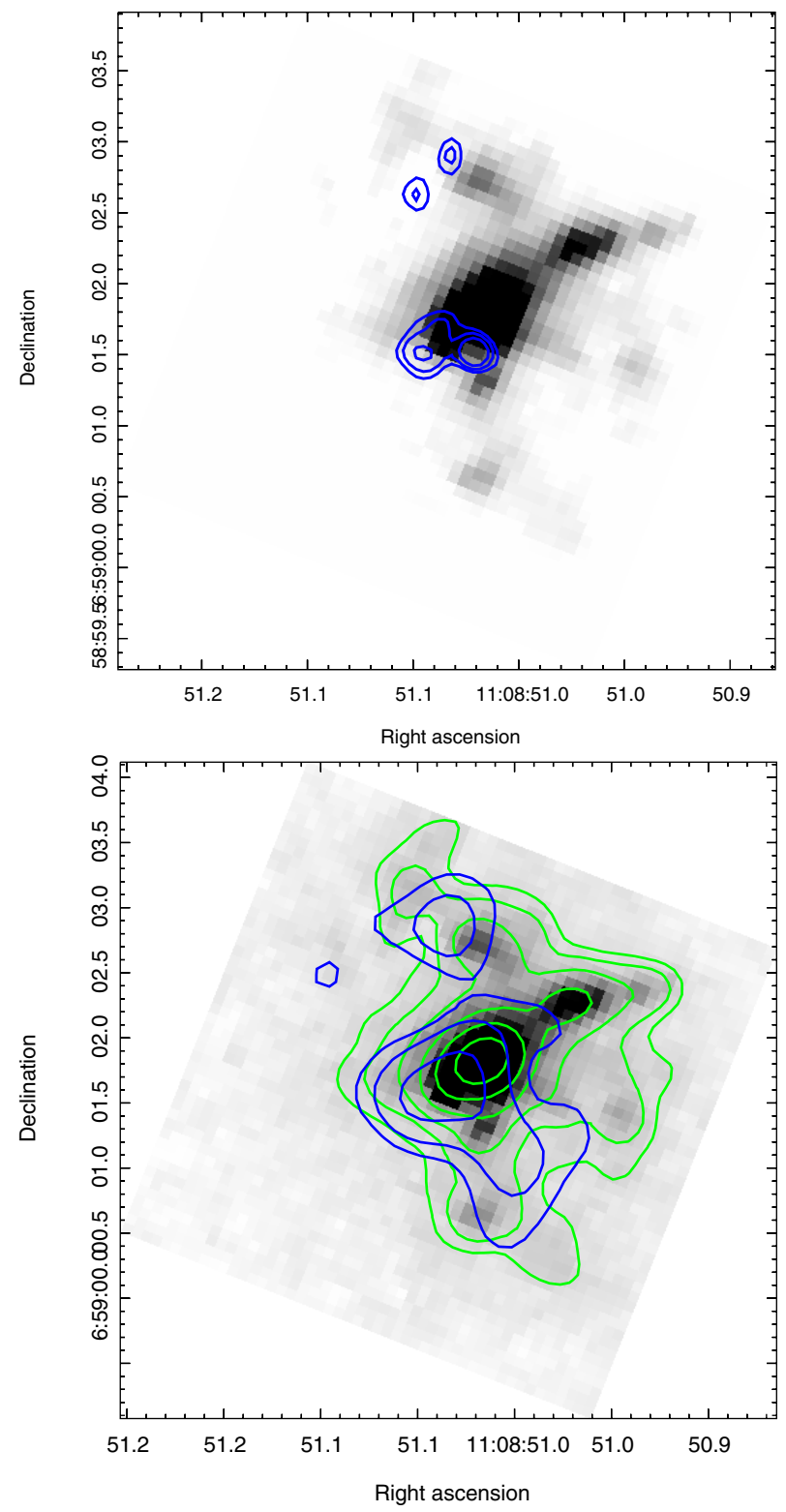

Fig. 4. HST $U$-band image of J1108+0659 overplot with the $8.5 \mathrm{GHz}$ VLA image (upper panel, HST in greys and VLA in contours) and with the $5.0 \mathrm{GHz}$ VLA image (lower panel, HST in greys and green contours and VLA in blue contours). The overplot with the $8.5 \mathrm{GHz}$ radio image shows the slight offset between the candidate radio core and the north-west core detected in the UV continuum. The south-east core is undetected in the UV continuum. The overplot with the $5.0 \mathrm{GHz}$ radio image clearly shows that the extended radio emission and the UV continuum are cospatial.

goal of these observations was to detect, in both objects, two radio cores to unambigously confirm the presence of a dual AGN. Our results can be summarized as follows:

- J1131-0204. We detect only one compact radio core, associated with the eastern optical/NIR nucleus of this system with a $5 \mathrm{GHz}$ luminosity of $L_{R}=6.5 \times 10^{28} \mathrm{erg} \mathrm{s}^{-1} \mathrm{~Hz}^{-1}$ typical of low luminosity AGN. For the second possible AGN in this system we can only provide a $5 \sigma$ upper limit on its $5 \mathrm{GHz}$ radio luminosity of $L_{R}=3.5 \times 10^{28} \mathrm{erg} \mathrm{s}^{-1} \mathrm{~Hz}^{-1}$. No X-ray emission is detected in either of the optical/NIR nuclei, even though the X-ray to [OIII] luminosity relation for type 2 AGN would yield $L_{2-10 \mathrm{kev}} \sim 10^{43} \mathrm{erg} \mathrm{s}^{-1}$ for each of the 
muclei. This is a clear indication that the AGN is strongly obscured by a Compton-thick screen.

- J1108+0659. This merging system is rather puzzling. From our EVN observations, we can only put upper limits on the radio luminosity of possible compact cores on the milliarcsecond scale. Using VLA observations we find a complex radio morphology on sub-arcsecond scale with a tentative core identification at $8.5 \mathrm{GHz}$. The core (component $\mathrm{C} 1$ ) has a flat radio spectrum and is slightly resolved, and its non detection in the EVN observations could be consistent with the sensitivity limit of our observations. Moreover, the position of this tentative radio core is offset $(\sim 0.2 \mathrm{arcsec})$ with respect to the optical/NIR and X-ray positions of the closest nucleus. Further radio observations that fill the gap in angular resolution between the $8.5 \mathrm{GHz}$ VLA and the $5 \mathrm{GHz}$ EVN observations are needed to establish the nature of the radio core candidate $\mathrm{C} 1$. Most of the extended sub-arcsecond scale radio emission is closely related to the UV continuum and with the [OIII] line emission, suggesting that it could come from intense star formation. Disentangling the AGNrelated from star formation-related radio emission, we derived $S F R \simeq 110 M_{\odot} \mathrm{yr}^{-1}$ that is consistent with what is expected from the total infrared luminosity, but an order of magnitude higher than implied by the UV luminosity, in turn implying large obscuration by dust.

Acknowledgements. The European VLBI Network is a joint facility of independent European, African, Asian, and North American radio astronomy institutes. Scientific results from data presented in this publication are derived from the following EVN project EB050. M.P.T. and E.P. are members of the MAGNA project (http://www.issibern.ch/teams/agnactivity/Home. html), and they gratefully acknowledge support of the International Space Science Institute (ISSI) in Bern, Switzerland. M.P.T. acknowledges support by the Spanish MINECO through grants AYA 2009-13036-C02-01 and AYA 201238491- C02-02, cofunded with FEDER funds. M.P.T. also acknowledges the hospitality of the Department of Theoretical Physics of the University of Zaragoza, where part of this work was done. The authors thank an anonymous referee for useful suggestions and comments that have improved the paper.

\section{References}

Beasley, A. J., \& Conway, J. E. 1995, in Very Long Baseline Interferometry and the VLBA, eds. J. A. Zensus, P. J. Diamond, \& P. J. Napier, ASP Conf. Ser., 82,327

Begelman, M. C., Blandford, R. D., \& Rees, M. J. 1980, Nature, 287, 307
Bianchi, S., Chiaberge, M., Piconcelli, E., Guainazzi, M., \& Matt, G. 2008, MNRAS, 386, 105

Bondi, M., \& Pérez-Torres, M.-A. 2010, ApJ, 714, L271

Bridle, A. H., \& Schwab, F. R. 1999, in Synthesis Imaging in Radio Astronomy

II, eds. G. B. Taylor, C. L. Carilli, \& R. A. Perley, ASP Conf. Ser., 180, 371 Colpi, M. 2014, Space Sci. Rev., 183, 189

Comerford, J. M., Griffith, R. L., Gerke, B. F., et al. 2009, ApJ, 702, L82

Dahlem, M., Weaver, K. A., \& Heckman, T. M. 1998, ApJS, 118, 401

Deane, R. P., Paragi, Z., Jarvis, M. J., et al. 2014, Nature, 511, 57

Escala, A., Larson, R. B., Coppi, P. S., \& Mardones, D. 2004, ApJ, 607, 765

Fu, H., Myers, A. D., Djorgovski, S. G., \& Yan, L. 2011a, ApJ, 733, 103

Fu, H., Zhang, Z.-Y., Assef, R. J., et al. 2011b, ApJ, 740, L44

Fu, H., Yan, L., Myers, A. D., et al. 2012, ApJ, 745, 67

Fu, H., Myers, A. D., Djorgovski, S. G., et al. 2015a, ApJ, 799, 72

Fu, H., Wrobel, J. M., Myers, A. D., Djorgovski, S. G., \& Yan, L. 2015b, ApJ, 815, L6

Gitti, M., Giroletti, M., Giovannini, G., Feretti, L., \& Liuzzo, E. 2013, A\&A, 557, L14

Hodge, J. A., Becker, R. H., White, R. L., Richards, G. T., \& Zeimann, G. R. 2011, AJ, 142, 3

Hou, L. G., Wu, X.-B., \& Han, J. L. 2009, ApJ, 704, 789

Hudson, D. S., Reiprich, T. H., Clarke, T. E., \& Sarazin, C. L. 2006, A\&A, 453, 433

Junkkarinen, V., Shields, G. A., Beaver, E. A., et al. 2001, ApJ, 549, L155

Komatsu, E., Smith, K. M., Dunkley, J., et al. 2011, ApJS, 192, 18

Komossa, S., Burwitz, V., Hasinger, G., et al. 2003, ApJ, 582, L15

Kormendy, J., \& Richstone, D. 1995, ARA\&A, 33, 581

Koss, M., Mushotzky, R., Treister, E., et al. 2011, ApJ, 735, L42

Liu, X., Greene, J. E., Shen, Y., \& Strauss, M. A. 2010a, ApJ, 715, L30

Liu, X., Shen, Y., Strauss, M. A., \& Greene, J. E. 2010b, ApJ, 708, 427

Liu, X., Civano, F., Shen, Y., et al. 2013, ApJ, 762, 110

Madau, P., \& Quataert, E. 2004, ApJ, 606, L17

Milosavljević, M., \& Merritt, D. 2001, ApJ, 563, 34

Moshir, M., Kopman, G., \& Conrow, T. A. O. 1992, IRAS Faint Source Survey, Explanatory supplement version 2

Nagar, N. M., Falcke, H., \& Wilson, A. S. 2005, A\&A, 435, 521

Newman, J. A., Cooper, M. C., Davis, M., et al. 2013, ApJS, 208, 5

Piconcelli, E., Vignali, C., Bianchi, S., et al. 2010, ApJ, 722, L147

Rodriguez, C., Taylor, G. B., Zavala, R. T., et al. 2006, ApJ, 646, 49

Schneider, D. P., Richards, G. T., Hall, P. B., et al. 2010, AJ, 139, 2360

Shen, Y., Liu, X., Greene, J. E., \& Strauss, M. A. 2011, ApJ, 735, 48

Shirazi, M., \& Brinchmann, J. 2012, MNRAS, 421, 1043

Smith, K. L., Shields, G. A., Bonning, E. W., et al. 2010, ApJ, 716, 866

Szomoru, A. 2008, in The role of VLBI in the Golden Age for Radio Astronomy, PoS(IX EVN Symposium) 040

Terashima, Y., \& Wilson, A. S. 2003, ApJ, 583, 145

Toomre, A., \& Toomre, J. 1972, ApJ, 178, 623

Ueda, Y., Hashimoto, Y., Ichikawa, K., et al. 2015, ApJ, 815, 1

Wang, J.-M., Chen, Y.-M., Hu, C., et al. 2009, ApJ, 705, L76

White, R. L., Becker, R. H., Helfand, D. J., \& Gregg, M. D. 1997, ApJ, 475, 479

Wrobel, J. M., Walker, R. C., \& Fu, H. 2014, ApJ, 792, L8 